\title{
The impact of age and BMI on oocyte maturation and embryo development.
}

\author{
Manal Taha Al-Obaidi', Huda Bahjat Mahdi², Estabraq Alwasiti ${ }^{\text {* }}$ \\ ${ }^{1}$ Institution for Infertility Diagnosis and Assisted Reproductive Technologies, University of Al-Nahrain, Baghdad, Iraq \\ ${ }^{2}$ Department of Clinical Reproductive Physiology, High Institution for Infertility Diagnosis and Assisted Reproductive \\ Technologies, University of Al-Nahrain, Baghdad, Iraq \\ ${ }^{3}$ Department of Biochemistry, College of Medical, University of Al-Nahrain, Baghdad, Iraq
}

\begin{abstract}
Background: Ovarian oogenesis is under the control of gonadotropins. In the human ovary, each fully grown oocyte resumes maturation in response to gonadotrophins. This process is completed after oocytes reach metaphase stage of second meiotic division (MII) stage. The aim of this prospective, clinical trial was to perceive the age and BMI effect on oocytes maturation and embryos development in women undergoing control ovarian hyperstimulation.

Method: A total of 40 infertile women undergoing ovarian hyperstimulation their age range between 18-40 y old were included in this study using two type of protocol: long agonist and antagonist.

Result: Significant negative correlation was observed between the age and the number of retrieved MII oocytes. Significant negatively effects of the BMI on the number of MII oocytes while significant positive correlation between the BMI and the fertilization rate was noticed.
\end{abstract}

Conclusion: There are negative effects of age and BMI on the oocytes number and quality.

Keywords: Oocyte maturation, Embryo development, Age, BMI.

Accepted on March 12, 2018

\section{Introduction}

The highest fertile capacity of women is in the age group between 20-24 y; after that a progressive decrease of fertility occurs with age [1]. The decline in female fertility begins several years before menopause, even with continual regular ovulatory cycles [2]. Fertility peaks when a woman is in her late teens and early twenties and begins to decline at age thirty, dropping more rapidly after age $35 \mathrm{y}$ then fertility plummets after age 40 and pregnancy after age 45 is rare [2].

It is well recognized that the fertility potential of women decreases with increasing age. The decline in female reproductive capacity with increasing age has two main causes: Gradual depletion of oocytes from the ovary, and a decrease in oocyte quality [3].

The reproductive organs of the human female exhibit a rate of ageing that is much faster than that of the other body systems, the same picture emerges from IVF studies where female age is undoubtedly the most significant factor influencing clinical outcome [4]. Ovarian functional decline with ageing has been so far extensively characterized in terms of gradual depletion of ovarian follicles and reduced ability to produce oocytes competent for fertilization and further development [5].

A fully developmentally competent oocyte is an oocyte that through oogenesis achieves the ability to resume meiosis, become fertilized with a single spermatozoa, decondense the sperm head, create two pronuclei $(\mathrm{PN})$, pass cleavage stages, undergo maternal to embryonic transition, reach the blastocyst stage, hatch, and establish a pregnancy that results in the birth of a healthy baby [3].

Higher risks of infertility have been found in both overweight and underweight women [6]. BMI is a calculated measurement that compares a person's weight and height and it is defined as the weight in kilograms divided by the square of the height in meters $\left(\mathrm{kg} / \mathrm{m}^{2}\right)$. The World Health Organization (WHO) and the National Institutes of Health (NIH) describe underweight as a $\mathrm{BMI}<18.5$, normal weight as BMI 18.5-24.9, overweight as BMI 25-29.9 and obesity as BMI>30 [7].

\section{Materials and Methods}

This Prospective clinical trial involved patient who were undergoing controlled ovarian hyperstimulation during period of study between August 2016 and February 2017 in outpatient department of high institution for infertility diagnosis and Assisted Reproductive Technologies, Al-Nahrain University, Baghdad-Iraq were recruited in this study.

The present study involved 40 Iraqi infertile women aged (18-39 y) who were undergoing controlled ovarian hyperstimulation. 
Full history and all details of protocols were taken from the patients and weight and height measured for Body Mass Index (BMI) calculation.

\section{Statistical analysis}

Statistical analysis was performed by using statistical package of science (SPSS); version 17.0 and Microsoft excel worksheet 2010 .

The numerical data analysis was done by using correlation, unpaired sample t-test for tables. The significance of the differences between values was considered statistically significant at the level of $(p<0.05)$. The descriptive measure of association in a correlational study is "correlation coefficient" (r) which ranges from $(-1)$ to $(+1)$.

If $\mathrm{r}=$-ve-- $>$ inverse association, if $\mathrm{r}=+\mathrm{ve}--\mathrm{-}+\mathrm{ve}$ association and if $\mathrm{r}=0-->$ no association.

Fertilization rate calculation was done by dividing the number of fertilized oocytes to the total number of MII oocytes that undergoing ICSI then the result multiplied to 100.

Fertilization rate $=$ (number of fertilized oocytes $/$ total number of MII oocytes that undergoing ICSI) $\times 100$.

\section{Basic steps in the ICSI}

The ICSI and embryo transfer process include stimulating the development of healthy ova in the ovaries, ova collection, sperms collection, as well as monitoring the fusion of the ova and selected sperm in the laboratory after intracytoplasmic injection by providing the suitable environment for fertilization and early embryo growth and finally followed by transferring the embryos into the uterus.

\section{Control ovarian hyperstimulation $(\mathrm{COH})$}

Two types of $\mathrm{COH}$ protocols are involved in this study:

The long GnRH agonist protocol: The long GnRH-agonist protocol for $\mathrm{COH}$ included down-regulation of pituitary gland with subcutaneous injection of GnRH-agonist $(0.1 \mathrm{mg} / \mathrm{d})$ that was begun on $21^{\text {st }} \mathrm{d}$ of the preceding menstrual cycle, then gonadotropin injection administered subcutaneously on the $2^{\text {nd }}$ or $3^{\text {rd }} \mathrm{d}$ of menstruation at different dosages according to the patient's age and previous response of ovulation induction. When at least three follicles had reached $18 \mathrm{~mm}$ in diameters HCG injection (10,000 IU) was used for triggering were applied 34-36 $\mathrm{h}$ prior to oocytes retrieval [8]. The antagonist protocol included administration of gonadotropin on the $2^{\text {nd }}$ or $3^{\text {rd }} \mathrm{d}$ of menstruation at different dosages according to the patient's age and previous response of ovulation induction then down-regulation of pituitary gland with SC injection of GnRH antagonist when follicular size reached $14 \mathrm{~mm}$ in diameter then triggering were applied 34-36 $\mathrm{h}$ prior to oocytes retrieval [9]. The $\mathrm{COH}$ was monitored by both serum E2 levels and transvaginal ultrasound [9].

Oocytes pickup (OPU): It is a procedure used in IVF to take out oocytes directly from the woman ovary prior to their liberate from ovarian follicles enabling fertilization outside the body [10]. It is performed under a guide of transvaginal ultrasound with an attached needle [10]. The oocytes are removed from the follicles through the needle connected to a suction device, and usually performed after 34-36 h of hCG injection, as the oocytes are entirely mature just prior to the rupture of follicles $[10,11]$.

Oocyte preparation and maturity assessment: In the ICSI laboratory, the retrieved oocyte-corona-cumulus complexes were immediately classified according to their maturity by the embryologist. The cumulus oophorus and the corona radiata were removed from oocytes within $3 \mathrm{~h}$ after follicular aspiration. After denudation of oocyte a rapid morphological evaluation by an inverted microscopic is performed, including evaluation of the zona pellucid, cytoplasm, and perivitelline space for any abnormality, and evaluation and grading according to nuclear maturity as metaphase II (MII), metaphase I (MI), or prophase I (GV). Oocyte nuclear maturity, as assessed by light microscopy, is assumed to be at the MII stage when the PBI is visible in the PVS and it is characteristic of metaphase of the second meiotic division, whereas the presence of an intracytoplasmic nucleus called the 'germinal vesicle' $(\mathrm{GV})$ which is a characteristic of prophase I of the first meiotic division. The oocytes that have neither a visible GV nor PBI these oocytes are generally classified as MI oocytes.

ICSI and embryo culture: ICSI was performed 3-6 h after oocyte recovery. After removal of cumulus and corona cells, these oocytes were examined under inverted microscope and only oocytes at MII stage that did not show obvious signs of degeneration were used for ICSI.

The injected oocytes were cultured at $37^{\circ} \mathrm{C}$ and were covered by mineral oil in IVF medium and incubated in an atmosphere of $5 \% \mathrm{O}_{2}, 5 \% \mathrm{CO}_{2}$ and $90 \% \mathrm{~N}_{2}$. The fertilization was assessed 16-20 h after ICSI. The normally fertilized oocytes were considered only for final embryo transfer. These were cultured for an additional $24-30 \mathrm{~h}$ at $37^{\circ} \mathrm{C}$ in fresh $\mathrm{CO}_{2}$-equilibrated IVF medium.

Embryo grading: After ICSI the fertilization and quality of embryo were assessed by the embryologist via the embryo grade. In brief, embryo grading on days 2 and 3 (48 and $72 \mathrm{~h}$ after oocytes pickup) was depend on number, size, and symmetry of blastomeres in addition to the percentage of fragmentation.

Embryos were graded morphologically as follows:

Grade 1, symmetrical with equal- sized blastomeres and $<10 \%$ extracellular fragmentation.

Grade 2, with unequal blastomeres and $<10 \%$ extracellular fragmentation.

Grade 3, unequal blastomeres with 10\%-50\% extracellular fragmentation

Grade 4, $>50 \%$ blastomeric fragmentation with uneven blastomeres [12]. 


\section{Results}

Table 1 illustrates the correlation of age with laboratory IVF parameters. Significant negative correlation was observed between the age and the number of MII oocytes that retrieved ( $\mathrm{p}$ value $=0.037, \mathrm{r}=-0.331$ ) while no significant correlation was detected between the age and all other laboratory IVF parameters.

As demonstrated in Table 2, significant negative effects of the BMI on the number of MII oocytes ( $\mathrm{p}$ value $=0.049, \mathrm{r}=-0.313$ ) while significant positive correlation between the BMI and the fertilization rate was noticed ( $p$ value $=0.042, r=0.323$ ), However no significant effects of the BMI on the other laboratory IVF parameters were observed.

Table 1. Correlation between the age with laboratory IVF parameters.

\begin{tabular}{lll}
\hline \multirow{2}{*}{ Parameters } & \multicolumn{2}{l}{ Age } \\
\cline { 2 - 3 } & R & P value \\
\hline Total oocyte NO. & -0.262 & 0.102 \\
\hline MII & -0.331 & $0.037^{*}$ \\
\hline MI & -0.088 & 0.588 \\
\hline GV & -0.035 & 0.829 \\
\hline Abnormal & 0.014 & 0.93 \\
\hline Fertilization rate & -0.124 & 0.445 \\
\hline No. of G1 ET & -0.299 & 0.061 \\
\hline
\end{tabular}

*Significant; MII: Metaphase II (mature oocytes); MI: Metaphase I (immature oocytes); GV: Germinal vesicle (prophase I stage), G1 ET: Grade 1 Embryo Transferred.

Table 2. Correlation of body mass index with IVF parameters.

\begin{tabular}{lll}
\hline \multirow{2}{*}{ Parameters } & \multicolumn{2}{l}{ BMI } \\
\cline { 2 - 3 } & $\mathbf{R}$ & $\mathbf{P}$ \\
\hline Total oocyte NO. & -0.283 & 0.077 \\
\hline MII & -0.313 & $0.049^{*}$ \\
\hline MI & -0.023 & 0.888 \\
\hline GV & 0.026 & 0.872 \\
\hline Abnormal & -0.19 & 0.239 \\
\hline Fertilization rate & 0.323 & $0.042^{*}$ \\
\hline No. of G1 ET & -0.136 & 0.402 \\
\hline
\end{tabular}

"Significant; MII: Metaphase II (mature oocytes); MI: Metaphase I (immature oocytes); GV: Germinal vesicle (prophase I stage); G1 ET: Grade 1 Embryo Transferred.

\section{Discussion}

The reproductive organs of the human female exhibit a rate of ageing that is much faster than that of the other body systems, the same picture emerges from IVF studies where female age is undoubtedly the most significant factor influencing clinical outcome [4]. Ovarian functional decline with ageing has been so far extensively characterized in terms of gradual depletion of ovarian follicles and reduced ability to produce oocytes competent for fertilization and further development [5].

In this study, Table 1 demonstrates a significant adverse effects of the age on the number of mature oocytes (MII), in addition the number of good quality embryo decrease as the age increase while no effect of age on the other IVF parameters.

Some studies shows that there is a significant effect of age on the expression of genes involved in cell cycle regulation (e.g. microtubule-based processes, chromatin assembly, M-phase of the meiosis) in oocytes in metaphase II (MII) from women undergoing IVF or ICSI treatment, these findings supported that meiosis and cell cycle regulation mechanisms may be affected by increasing age of the woman [13]. In addition, at various developmental stages, follicles behave differently in response to factors promoting follicular cell proliferation, growth, differentiation and apoptosis and very few reach ovulation [14].

Thus, based on the above observations the analysis of the molecular and cellular aspects of follicle ageing would require careful consideration of some main points. First, oocytes and granulosa cells of primordial follicles might remain in a 'resting' phase for a long time, thus behaving as post-mitotic cells which can be required to start growing after 10-50 y. Second, both primordial and growing follicles become exposed to environmental factors related to the ageing of the ovarian somatic compartment. Third, the development of a competent oocyte intimately depends on the cross-talk between all compartments in the ovary.

The present data illustrate that the fertilization rate was not influenced by the increasing age of the women in ICSI. In addition our study shows a tendency towards decreased number of G1 embryo with increasing maternal age further supports that the $2^{\text {nd }}$ meiotic division may be influenced by age, since a recent publication comparing two age groups reports increased time to $2^{\text {nd }}$ polar body extrusion after ICSI in aged women as compared to younger women [15]. In contrary other studies has been found that embryo quality is not affected by women's age [16].

Obesity has become a major health problem across the world. In women, it is known to cause anovulation, sub-fecundity, increased risk of fetal anomalies and miscarriage rates [17]. However, in women going for assisted reproduction the effects of obesity on egg quality, embryo quality, clinical pregnancy, live birth rates are controversial. Reduced fecundity in obese women is probably related to multiple factors including aberrations in endocrine and metabolic functions that in turn can affect follicular growth, implantation and development of a clinical pregnancy [17].

The results presented in this study assumed an adverse effect of BMI on the oocytes maturation and fertilization rate while no effects on the number of retrieved oocytes, immature oocytes and G1 transferred embryos, as shown in Table 2. The retrieved mature oocytes and fertilization rate decrease with 
increasing the BMI and confirm the findings of published reports with some investigator $[18,19]$. This result was already found in experiments conducted in mice fed with a high fat obesogenic diet, reporting oocytes with delayed maturation and decreased developmental competence in addition it has been demonstrated that these problems with oocyte function are a direct result of a mitochondrial dysfunction. In fact, a high fat obesogenic diet leads to abnormalities of mitochondrial morphology, mitochondrial distribution within the oocyte, metabolism and spindle formation within the oocytes [19]. While the other investigator explains it as obese women required increased doses of gonadotropin for inducing ovulation or stimulating the ovaries to obtain mature oocytes and impaired ovarian responsiveness to gonadotropin stimulation were reported to be associated with obesity [20].

Several investigators have found a decrease in the number of retrieved oocytes in overweight and obese compared with normal weight women, most likely due to decreased response to control ovarian hyperstimulation with increased BMI, whereas others have found no difference [17,20-22]. Other authors have noted an increased frequency of cycle cancellation due to insufficient follicular development [22,23].

\section{References}

1. Fritz MS. Clinical gynecologic endocrinology and infertility (8th ed). Lippincott Williams \& Wilkins, Philadelphia, USA 2011; 1138-1190.

2. Rizk Botros RMB, Garcia-Velasco Juan A, Sallam Hassan N. Infertility and assisted reproduction. Cambridge University Press, New York 2008; 643.

3. Grøndahl ML, Christiansen SL, Kesmodel US, Agerholm IE, Lemmen JG, Lundstrøm P. Effect of women's age on embryo morphology, cleavage rate and competence-A multicenter cohort study. PLoS One 2017; 12: 12.

4. Te Velde ER, Pearson PL. The variability of female reproductive ageing. Hum Reprod Update 2002; 8: 141-154.

5. Broekmans FJ, Knauff EAH, Te Velde ER, Macklon NS, Fauser BC. Female reproductive ageing: current knowledge and future trends. Trends Endocrinol Metab 2007; 18: 58-65.

6. Wittemer C, Ohl J, Bailly M, Bettahar-Lebugle K, Nisand I. Does body mass index of infertile women have an impact on IVF procedure and outcome? J Assist Reprod Genet 2000; 17: 547-552.

7. Seli E. Infertility. Wiley-Blackwell, Hoboken, New Jersey, United States 2011; 201.

8. Kamath M, Deepti M. Unexplained infertility: An approach to diagnosis and management. Curr Med Issues 2016; 14: 94.

9. Shrestha D, La X, Feng HL. Comparison of different stimulation protocols used in in vitro fertilization: a review. Ann Transl Med 2015; 3: 137.

10. Gardner D. In vitro fertilization: a practical approach. Informa Healthcare; 2007; 507.
11. Sauer MV. Assisted reproductive technology. Western J Med 2015; 161: 164-165.

12. Coughlan C, Ledger B, Ola B. In-vitro fertilization. Obstetr Gynaecol Reprod Med 2011; 21: 303-310.

13. Grøndahl ML, Christiansen SL, Kesmodel US, Agerholm IE, Lemmen JG, Lundstrøm P. Effect of women's age on embryo morphology, cleavage rate and competence-A multicenter cohort study. PLoS One 2017; 12: 12.

14. Weber GM, Moore AB, Sullivan CV. In vitro actions of insulin-like growth factor-I on ovarian follicle maturation in white perch (Morone americana). Gen Comp Endocrinol 2007; 151: 180-187.

15. Liu Y, Chapple V, Roberts P, Ali J, Matson P. Time-lapse videography of human oocytes following intracytoplasmic sperm injection: Events up to the first cleavage division. Reprod Biol 2014; 14: 249-256.

16. Stensen MH, Tanbo T, Storeng R, Åbyholm T, Fèdorcsak P. Routine morphological scoring systems in assisted reproduction treatment fail to reflect age-related impairment of oocyte and embryo quality. Reprod BioMed Online 2010; 21: 118-125.

17. Fedorcsák P, Dale PO, Storeng R, Ertzeid G, Bjercke S, Oldereid N. Impact of overweight and underweight on assisted reproduction treatment. Hum Reprod 2004; 19: 2523-2528.

18. Christensen MW, Ingerslev HJ, Degn B, Kesmodel US. Effect of female body mass index on oocyte quantity in fertility treatments (IVF): Treatment cycle number is a possible effect modifier. A register-based cohort study. PLoS One 2016; 11: e0163393.

19. Sarais V, Pagliardini L, Rebonato G, Papaleo E, Candiani $\mathrm{M}$, Viganò $\mathrm{P}$. A comprehensive analysis of body mass index effect on in vitro fertilization outcomes. Nutrients 2016; 8: 109.

20. Cui N, Wang H, Wang W, Zhang J, Xu Y, Jiang L. Impact of body mass index on outcomes of in vitro fertilization/ intracytoplasmic sperm injection among polycystic ovarian syndrome patients. Cell Physiol Biochem 2016; 39: 1723-1734.

21. Lintsen AME, Pasker-de Jong PCM, de Boer EJ, Burger CW, Jansen CAM, Braat DDM. Effects of subfertility cause, smoking and body weight on the success rate of IVF. Hum Reprod 2005; 20: 1867-1875.

22. Van Swieten ECAM, Van Der Leeuw-Harmsen L, Badings EA, Van Der Linden PJQ. Obesity and clomiphene challenge test as predictors of outcome of in vitro fertilization and intracytoplasmic sperm injection. Gynecol Obstetr Invest 2005; 59: 220-224.

23. Dokras A, Baredziak L, Blaine J, Syrop C, Vanvoorhis BJ, Sparks A. Obstetric outcomes after in vitro fertilization in obese and morbidly obese women. Obstet Gynecol 2006; 108: 61-69.

\section{*Correspondence to}

Estabraq Alwasiti 
The impact of age and BMI on oocyte maturation and embryo development

Department of Biochemistry

College of Medical

University of Al-Nahrain

Baghdad

Iraq 